\title{
Helping patients with lung cancer choose between surgery or stereotactic body radiotherapy: the importance of the patient experience
}

\author{
Michael MacManus ${ }^{1,2}$, Richard De Abreu Lourenco ${ }^{3}$, Fiona Hegi-Johnson ${ }^{1,2}$ \\ ${ }^{1}$ Peter MacCallum Cancer Centre, Melbourne, Australia; ${ }^{2}$ The Sir Peter MacCallum Department of Oncology, University of Melbourne, Melbourne, \\ Australia; ${ }^{3}$ Centre for Health Economics Research and Evaluation, University of Technology Sydney, Sydney, Australia \\ Correspondence to: Professor Michael P. MacManus. Department of Radiation Oncology, Peter MacCallum Cancer Centre, 305 Grattan Street, \\ Melbourne, Victoria 3000, Australia. Email: Michael.macmanus@petermac.org. \\ Provenance: This is an invited article commissioned by the Editorial Office, Fournal of Thoracic Disease. \\ Comment on: Takeda A, Sanuki N, Tsurugai Y, et al. Questionnaire survey comparing surgery and stereotactic body radiotherapy for lung cancer: \\ lessons from patients with experience of both modalities. J Thorac Dis 2019;11:2479-89.
}

Submitted Sep 04, 2019. Accepted for publication Sep 26, 2019.

doi: $10.21037 /$ jtd.2019.10.13

View this article at: http://dx.doi.org/10.21037/jtd.2019.10.13

Stereotactic body radiotherapy (SBRT) has been rapidly transforming the management of patients with small primary and secondary lung tumours who are either considered unfit for surgery, or who are of borderline fitness for such widely used surgical approaches as lobectomy or segmental resection. The recently published CHISEL trial has confirmed the superiority of SBRT over conventional conformal radiotherapy in patients who are not surgical candidates, both for local disease control and overall survival (1). As a consequence of the high rates of local control achieved with SBRT in medically inoperable lung cancers, and the low rates of toxicity reported for appropriately selected patients, treated with modern dose and fractionation schedules, delivered with appropriate immobilization, employing high quality treatment planning protocols and using technically advanced modern linear accelerators, SBRT is being considered as an alternative to surgical resection in an increasing number of cases worldwide. Clinical outcomes for surgery and SBRT are currently being compared in randomized controlled trials (RCTs).

It is important that trials comparing such contrasting treatments should consider the "patient experience" of the two modalities, which are likely to be vastly different. It is in this regard that the data published by Takeda et al. (2) in this journal can provide information that is extremely helpful, both to patients and clinicians. When a patient is asked to choose between two very different treatment strategies for the same cancer, disease control and survival are critically important but they are not the only considerations. The patient experience, during and after the treatment can also be powerful factors that inform decision making. There are several published studies that have presented patientreported quality of life data after SBRT (3-7), including a report from the ROSEL (8) trial and after surgery (9-11) but direct comparative studies are lacking. Because quality of life measures are so heavily influenced by parameters other than treatment, including co-morbidities and socioeconomic factors, non-randomised comparisons are subject to bias, including from the selection of cohorts that are not directly comparable and from the unconscious bias of investigators.

With respect to disease control and survival, no successfully-completed RCTs have yet been published that have compared SBRT and surgery in medically operable patients. However, investigators in the STARS and ROSEL trials combined data from their prematurely terminated RCTs, both of which failed to accrue sufficient patients, and performed analyses as if their patients had been recruited to the same trial. Chang and colleagues published the results in Lancet Oncology (12). Eligible patients had clinical stage T1-2a $(<4 \mathrm{~cm})$, NOM0, operable NSCLC and were randomly assigned in a 1:1 ratio to SBRT or lobectomy with mediastinal lymph node dissection or sampling. Only 
58 patients were enrolled, and median follow-up was 40.2 months. Estimated 3 years overall survival was $95 \%$ in the SABR group compared with $79 \%$ in the surgery group (hazard ratio HR 0.14; log-rank $\mathrm{P}=0.037$ ) and recurrencefree survival at 3 years was $86 \%$ for SABR versus $80 \%$ in the surgery group (HR 0.69; log-rank $\mathrm{P}=0.54$ ). These provocative data suggest that, at least for some subsets of patients, surgery and SBRT may achieve early outcomes that are comparable. This study did not include useful comparisons of the patient experiences with the two treatment modalities.

The methodology used in the approach of Chang and colleagues in their Lancet Oncology paper has been much discussed and often criticised (13-16), especially from within the surgical community. It is likely that some advocates of SBRT have overstated the significance of this publication and have asserted that it can be concluded that SBRT is superior to surgery based on these data $(17,18)$. On the other hand, despite their limitations, these controversial data remain the only relevant published "randomized" information so far available that provide a direct comparison of survival outcomes. There is increasingly wide agreement that these two modalities should be compared in medically operable patients in well designed and adequately powered randomized trials designed to compare survival, disease control and toxicity. The STABLE-MATES trial, which is randomising patients at a higher risk of perioperative complications to either SABR or sub-lobar resection, will hopefully be able to complete recruitment by employing a novel strategy of "pre-randomisation" to minimise drop-out between the two arms. Drop-out is a recognised problem in randomised trials where two widely different treatment strategies are compared and can sometimes be due to patient perception but may also reflect decision making and referrer bias.

\section{Patient decision making: what matters to patients?}

Whereas there is a great deal of information available on the survival and disease control outcomes for large cohorts of patients treated with either surgery or SBRT, there is surprisingly little comparative information available in the literature on patient experiences and preferences for each of these two complementary and increasingly competing modalities. Patient preferences will become increasingly important as SBRT becomes more widely available and utilized and as patients gain more information about this modality as a potential alternative to surgery in an increasing number of clinical scenarios.

In their article Takeda and colleagues provide real-world information on the actual experiences of patients managed with each of these very different modalities. For a group of patients treated with SBRT between 2005 and 2017, the authors identified a group who had previously undergone surgery for lung cancer. These patients were asked to complete a questionnaire that sought information on a range of relevant factors, including stress, adverse events and satisfaction after each of the two modalities around and after the time of treatment. The participants were also asked about treatment decision-making for a range of hypothetical scenarios. The authors identified 149 patients of whom 52 completed the study. Participating patients were relatively elderly (median age 76). When asked if they were more satisfied with SBRT or surgery, patients overwhelmingly preferred SBRT (27 of those expressing a preference preferred SBRT comparted to only 3 for surgery). When presented with a hypothetical scenario with equivalent expected outcomes for SBRT or surgery, the great majority (38/52) selected SBRT. Even when the expected outcomes were presented as $20 \%$ worse for SBRT than surgery, 14 subjects still selected SBRT, 12 chose surgery and 16 were unable to decide.

Given the necessarily invasive nature of surgical approaches, the often painful post-operative recovery period and the exposure of patients to risks of perioperative infection and bleeding, it is not altogether surprising that the experience of SBRT, which is a relatively brief and non-invasive outpatient procedure associated, in the great majority of cases associated with mild to minimal toxicity, would be preferred by most patients who have personally experienced both modalities.

As the authors indicate there are a number of limitations to their study. Patients undergoing sequential treatment, and receiving SBRT for recurrence may perceive that the surgery was ineffective, thereby biasing them against thinking of this as effective treatment. Similarly, such patients may be more favourably disposed to SBRT as an option-perceiving it as being required to "rescue" them from "ineffective" surgery. The majority of patients $(75 \%)$ had an open thoracotomy; the increasing use of minimally invasive procedures should in future serve to reduce the physical discomfort and pain associated with the surgical resection, as well as reducing the risk of perioperative complications. It would also have been helpful to include a cohort of patients from a thoracic surgical centre to 
minimise the influence of patient selection and referrer bias within the study. Another factor to be considered, is the risk of mortality in the perioperative period $(19,20)$, especially in elderly patients after surgical resection. Patients who did not survive the post-operative period will clearly not have completed the questionnaires.

The inclusion of a simple decision choice question within the survey provided initial insights into patient views. However, it is unsurprising that this echoes the views expressed in the patient satisfaction ratings. Moreover, the format used cannot be used to inform decision-makers as to which aspects of surgery or SBRT most influence patient preferences. For this purpose, comprehensive, formally designed discrete-choice experiments are a more effective means of teasing out the complex interplay of factors that influence patient choice in the setting of lung cancer (21).

Intriguingly, when patients who have not experienced both forms of treatment are surveyed, overall survival rises in importance, and appears to be the overriding consideration for the majority of patients (22). Although there is likely to remain a subset of patients who rely almost solely on expert opinion for the treatment decision, with the increasing availability of internet-based information and social media, patients come to us with more and more information than ever before. It behoves us to ensure that the information and advice they receive from us is accessible and clear. Patients require an unbiased and objective description of the true likely impact of treatment upon quality of life and survival.

\section{Conclusions}

As the authors rightly note, there is an unmet need for high-quality, prospective clinical evaluation in this area. As far as possible, we would encourage all investigators of clinical trials to include patient reported outcomes, and health economics methodologies into their clinical trials to ensure that their results are not only scientifically valid, but meaningful within the specific social, cultural and economic community of our patients. These aspects are not mere adjuncts, added on as after-thoughts to a trial, but essential for us to advocate for the increased resources that promising new technologies, such as robotic and videoassisted thoracic surgery (VATS), novel radiation therapies, interventional oncology and combined modality approaches with immunotherapy, will require. Takada $e t$ al. are to be commended for a thoughtful analysis of a unique data-set that provides a model to assess the true benefits of clinical innovation in the future through the integration of these priorities in future prospective trials.

\section{Acknowledgments}

None.

\section{Footnote}

Conflicts of Interest: The authors have no conflicts of interest to declare.

Ethical Statement: The authors are accountable for all aspects of the work in ensuring that questions related to the accuracy or integrity of any part of the work are appropriately investigated and resolved.

\section{References}

1. Ball D, Mai GT, Vinod S, et al. Stereotactic ablative radiotherapy versus standard radiotherapy in stage 1 nonsmall-cell lung cancer (TROG 09.02 CHISEL): a phase 3 , open-label, randomised controlled trial. Lancet Oncol 2019;20:494-503.

2. Takeda A, Sanuki N, Tsurugai Y, et al. Questionnaire survey comparing surgery and stereotactic body radiotherapy for lung cancer: lessons from patients with experience of both modalities. J Thorac Dis 2019;11:2479-89.

3. Sun V, Kim JY, Williams AC, et al. Quality of life and symptoms following stereotactic body radiotherapy in early-stage lung cancer patients. J Community Support Oncol 2014;12:407-14.

4. Lagerwaard FJ, Aaronson NK, Gundy CM, et al. Patient-reported quality of life after stereotactic ablative radiotherapy for early-stage lung cancer. J Thorac Oncol 2012;7:1148-54.

5. van der Voort van Zyp NC, Prévost JB, van der Holt B, et al. Quality of life after stereotactic radiotherapy for stage I non-small-cell lung cancer. Int J Radiat Oncol Biol Phys 2010;77:31-7.

6. Videtic GM, Reddy CA, Sorenson L. A prospective study of quality of life including fatigue and pulmonary function after stereotactic body radiotherapy for medically inoperable early-stage lung cancer. Support Care Cancer 2013;21:211-8.

7. Videtic GM. Early-Stage Lung Cancer, Surgery, and Stereotactic Body Radiation Therapy: Quality of Life. Int 
J Radiat Oncol Biol Phys 2016;96:927-30.

8. Louie AV, van Werkhoven E, Chen H, et al. Patient reported outcomes following stereotactic ablative radiotherapy or surgery for stage IA non-small-cell lung cancer: Results from the ROSEL multicenter randomized trial. Radiother Oncol 2015;117:44-8.

9. Kenny PM, King MT, Viney RC, et al. Quality of life and survival in the 2 years after surgery for non small-cell lung cancer. J Clin Oncol 2008;26:233-41.

10. Poghosyan H, Sheldon LK, Leveille SG, et al. Healthrelated quality of life after surgical treatment in patients with non-small cell lung cancer: a systematic review. Lung Cancer 2013;81:11-26.

11. Gazala S, Pelletier JS, Storie D, et al. A systematic review and meta-analysis to assess patient-reported outcomes after lung cancer surgery. ScientificWorldJournal 2013;2013:789625.

12. Chang JY, Senan S, Paul MA, et al. Stereotactic ablative radiotherapy versus lobectomy for operable stage I nonsmall-cell lung cancer: a pooled analysis of two randomised trials. Lancet Oncol 2015;16:630-7.

13. Cao C, D'Amico T, Demmy T, et al. Surgery versus SABR for resectable non-small-cell lung cancer. Lancet Oncol 2015;16:e370-1.

14. Dearman C, van As N, Crellin A, et al. Surgery versus SABR for resectable non-small-cell lung cancer. Lancet Oncol 2015;16:e373-4.

15. Hamaji M, Groth SS, Sugarbaker DJ, et al. Surgery versus SABR for resectable non-small-cell lung cancer. Lancet Oncol 2015;16:e372.

Cite this article as: MacManus M, De Abreu Lourenco R, Hegi-Johnson F. Helping patients with lung cancer choose between surgery or stereotactic body radiotherapy: the importance of the patient experience. J Thorac Dis 2019;11(11):4404-4407. doi: 10.21037/jtd.2019.10.13
16. Nieder C, Andratschke NH, Guckenberger M. A pooled analysis of stereotactic ablative radiotherapy versus lobectomy for operable stage I non-small cell lung cancer: is failure to recruit patients into randomized trials also an answer to the research question? Ann Transl Med 2015;3:148.

17. Meyers BF, Puri V, Broderick SR, et al. Lobectomy versus stereotactic body radiotherapy for stage I non-small cell lung cancer: Post hoc analysis dressed up as level-1 evidence? J Thorac Cardiovasc Surg 2015;150:468-71.

18. Rosen JE, Salazar MC, Wang Z, et al. Lobectomy versus stereotactic body radiotherapy in healthy patients with stage I lung cancer. J Thorac Cardiovasc Surg 2016;152:44-54.e9.

19. Stokes WA, Bronsert MR, Meguid RA, et al. PostTreatment Mortality After Surgery and Stereotactic Body Radiotherapy for Early-Stage Non-Small-Cell Lung Cancer. J Clin Oncol 2018;36:642-51.

20. Rusthoven CG, Palma DA, Senan S, et al. The Head Start Effect: Will Acute and Delayed Postoperative Mortality Lead to Improved Survival with Stereotactic Body Radiation Therapy for Operable Stage I Non-Small-Cell Lung Cancer? J Clin Oncol 2017;35:1749-51.

21. Clark MD, Determann D, Petrou S, et al. Discrete choice experiments in health economics: a review of the literature. Pharmacoeconomics 2014;32:883-902.

22. Tong BC, Wallace S, Hartwig MG, et al. Patient Preferences in Treatment Choices for Early-Stage Lung Cancer. Ann Thorac Surg 2016;102:1837-44. 